QUARTERLY OF APPLIED MATHEMATICS

VOLUME LXIX, NUMBER 4

DECEMBER 2011, PAGES 787-806

S $0033-569 X(2011) 01228-5$

Article electronically published on July 1, 2011

\title{
THERMODYNAMICS OF A NON-SIMPLE HEAT CONDUCTOR WITH MEMORY
}

\author{
BY \\ GIOVAMBATtiSta AMENDOLA (Dipartimento di Matematica Applicata "U. Dini", via F. \\ Buonarroti 1c, 56127-Pisa, Italy), \\ MAURO FABRIZIO (Dipartimento di Matematica, Piazza di Porta S. Donato 5, 40127-Bologna, \\ Italy), \\ AND \\ MURROUGH GOLDEN (School of Mathematical Sciences, Dublin Institute of Technology, Kevin \\ Street, Dublin 8, Ireland)
}

\begin{abstract}
A generalization is given of the linearized constitutive equation, proposed by Gurtin and Pipkin for the heat flux in a rigid heat conductor, which includes the effects of both the histories of the temperature gradient $\mathbf{g}$ and of $\nabla \mathbf{g}$. This new contribution yields a non-simple material, for which the Second Law of Thermodynamics assumes a modified form, characterized by an extra flux, depending on the material. Some standard free energy functionals are adapted to these new materials, including an explicit formula for the minimum free energy.
\end{abstract}

1. Introduction. A generalization of the linearized constitutive equation for the heat flux, proposed by Gurtin and Pipkin 18 is given in this paper, influenced by the work of Guyer and Krumhansl [19]. Using kinetic theory, they obtain a generalization of Cattaneo-Maxwell's equation containing new terms with second gradients of $\mathbf{q}$.

We consider a new constitutive equation with memory for the heat flux, which includes not only the effects of the history of the temperature gradient $\mathbf{g}$, but also the history of $\nabla \cdot \nabla \mathrm{g}$. This choice yields a non-simple material, for which the classical Second Law of Thermodynamics, expressed by the Clausius-Duhem inequality, must be modified by means of a different definition of internal entropy power or by considering the presence of an extra flux.

Received May 11, 2010.

2010 Mathematics Subject Classification. Primary 80A20, 74F05.

Key words and phrases. Memory effects, heat conduction.

The work of the first two authors was performed with support from the Italian C.N.R. and M.I.U.R.

The research of the third author was supported by the Dublin Institute of Technology.

E-mail address: amendola@dma.unipi.it

E-mail address: fabrizio@dm.unibo.it

E-mail address: murrough.golden@dit.ie 
We study the compatibility of the new non-local constitutive equation with the Second Law and derive restrictions on the kernels. Under these restrictions one can study free energies related to the non-local constitutive equation for the heat flux.

The layout of the paper is as follows. In Section 2, some constitutive equations for the heat flux are examined in a generalized linear theory of rigid heat conductors with memory effects. In Section 3, the classical form of the Second Law of Thermodynamics is considered for simple materials in order to introduce an appropriate modification for non-simple materials. In Section 4, after introducing states and processes for non-simple conductors, the linearized form of the Second Law is deduced for these materials. The thermodynamic restrictions on the constitutive equation for the heat flux are then derived. In Section 5, two free energies already introduced for viscoelastic materials are adapted to our non-simple rigid heat conductors. In Section 6, we derive an explicit expression for the minimum free energy of these materials.

2. Preliminaries. Gurtin and Pipkin developed [18] a general theory of heat conduction for non-linear materials with memory, based on the work of Coleman [5]. A major objective of this formulation was to avoid the infinite speed of propagation of thermal disturbances of the classical theory, characterized by Fourier's law

$$
\mathbf{q}(\mathbf{x}, t)=-\kappa \mathbf{g}(\mathbf{x}, t)
$$

where $\mathbf{q}$ denotes the heat flux and $\mathbf{g}=\nabla \vartheta$ is the temperature gradient, both of which depend on the position vector $\mathbf{x}$ and the time $t$. The scalar quantity $\kappa$ is the conductivity, which must be positive. The linearization of such a theory for isotropic media, also considered in [18, gives for the heat flux the following constitutive equation:

$$
\mathbf{q}(\mathbf{x}, t)=-\int_{0}^{+\infty} a(s) \mathbf{g}^{t}(\mathbf{x}, s) d s
$$

This expresses the heat flux $\mathbf{q}$ as a linear functional of the history of the temperature gradient $\mathbf{g}$ up to time $t, \mathbf{g}^{t}(\mathbf{x}, s)=\mathbf{g}(\mathbf{x}, t-s) \forall s \in \mathbf{R}^{+} \equiv[0,+\infty)$. The authors observed that Fourier's law is not a particular case of their functional, unless a constant temperature gradient has been imposed on the body for all past times. Under this assumption, $\mathbf{g}^{t}(\mathbf{x}, s)=\mathbf{g}(\mathbf{x}) \forall s \in \mathbf{R}^{+}$and (2.2) reduces to

$$
\mathbf{q}(\mathbf{x}, t)=-\kappa \mathbf{g}(\mathbf{x}, t), \quad \kappa=\int_{0}^{+\infty} a(s) d s
$$

They noted that their relation yields a generalization of the Cattaneo-Maxwell equation, previously introduced in a pioneering paper [3], which sought to avoid the propagation of heat at an infinite speed. To show this, they considered the Cattaneo-Maxwell 
relation 11 written in the form

$$
\dot{\mathbf{q}}(\mathbf{x}, t)+\sigma \mathbf{q}(\mathbf{x}, t)=-\kappa \sigma \mathbf{g}(\mathbf{x}, t)
$$

and observed that this expression is the special case of (2.2) corresponding to the kernel

$$
a(s)=\kappa \sigma e^{-\sigma s} .
$$

To prove this it is enough to replace the kernel $a(s)$ with (2.5) in (2.2), that is,

$$
\mathbf{q}(\mathbf{x}, t)=-\int_{0}^{+\infty} \kappa \sigma e^{-\sigma s} \mathbf{g}^{t}(\mathbf{x}, s) d s,
$$

and to check that this relation and its time derivative (after an integration by parts) satisfy (2.4).

We rewrite the Cattaneo-Maxwell relation in its original form:

$$
\tau \dot{\mathbf{q}}(\mathbf{x}, t)+\mathbf{q}(\mathbf{x}, t)=-\kappa \mathbf{g}(\mathbf{x}, \mathbf{t}) .
$$

Guyer and Krumhansl [19] (see also Cimmelli and Frischmuth [4]), with a suitable approximation, obtain from kinetic theory the following generalization of the CattaneoMaxwell equation:

$$
\tau \dot{\mathbf{q}}(\mathbf{x}, t)+\mathbf{q}(\mathbf{x}, t)=-k \nabla \vartheta(\mathbf{x}, t)+\alpha[\nabla \cdot \nabla \mathbf{q}(\mathbf{x}, t)+2 \nabla \nabla \cdot \mathbf{q}(\mathbf{x}, t)],
$$

where $\tau$ is the relaxation time, $k>0$ is the heat conduction and $\alpha$ is a suitable coefficient. An argument similar to that used by Guyer and Krumhansl (see also 4]) yields the new equation

$$
\tau \dot{\mathbf{q}}(\mathbf{x}, t)+\mathbf{q}(\mathbf{x}, t)=-h_{1} \mathbf{g}(\mathbf{x}, t)+h_{2} \nabla \cdot \nabla \mathbf{g}(\mathbf{x}, t),
$$

where, besides the effect of $\mathbf{g}$, we also have the contribution of the divergence of its gradient $\nabla \mathbf{g}$. This new constitutive equation is equivalent, in the Gurtin-Pipkin sense, to

$$
\mathbf{q}(\mathbf{x}, t)=-\int_{0}^{+\infty} \frac{h_{1}}{\tau} e^{-\gamma s} \mathbf{g}^{t}(\mathbf{x}, s) d s+\nabla \cdot \int_{0}^{+\infty} \frac{h_{2}}{\tau} e^{-\gamma s} \nabla \mathbf{g}^{t}(\mathbf{x}, s) d s, \gamma=\frac{1}{\tau} .
$$

In fact, its time derivative, taking account of $\dot{\mathbf{g}}^{t}(s)=-\frac{d}{d s} \mathbf{g}^{t}(s)$ and integrating by parts, yields

$$
\begin{aligned}
\dot{\mathbf{q}}(\mathbf{x}, t)= & \frac{h_{1}}{\tau} \int_{0}^{+\infty} e^{-\gamma s} \frac{d}{d s} \mathbf{g}^{t}(\mathbf{x}, s) d s-\nabla \cdot \frac{h_{2}}{\tau} \int_{0}^{+\infty} e^{-\gamma s} \frac{d}{d s} \nabla \mathbf{g}^{t}(\mathbf{x}, s) d s \\
=\gamma & {\left[\int_{0}^{+\infty} \frac{h_{1}}{\tau} e^{-\gamma s} \mathbf{g}^{t}(\mathbf{x}, s) d s-\nabla \cdot \int_{0}^{+\infty} \frac{h_{2}}{\tau} e^{-\gamma s} \nabla \mathbf{g}^{t}(\mathbf{x}, s) d s\right] } \\
& -\frac{1}{\tau}\left[h_{1} \mathbf{g}(\mathbf{x}, t)-h_{2} \nabla \cdot \nabla \mathbf{g}(\mathbf{x}, t)\right] \\
=\frac{1}{\tau} & {\left[-\mathbf{q}(\mathbf{x}, t)-h_{1} \mathbf{g}(\mathbf{x}, t)+h_{2} \nabla \cdot \nabla \mathbf{g}(\mathbf{x}, t)\right], }
\end{aligned}
$$

\footnotetext{
${ }^{1}$ The exact expression proposed by Cattaneo for the heat flux is

$$
\sigma \dot{\mathbf{q}}+\varkappa \mathbf{q}=-\varkappa^{2} \nabla \vartheta
$$

This form has the advantage over (2.4), adapted by Gurtin and Pipkin, that one can recover Fourier's law immediately from this form by putting $\sigma=0$. This was important for Cattaneo, who was very conscious of the importance of the Fourier relation, in particular because of its continuous use in technical applications. Therefore, we use such an expression in the form (2.7), where $\tau=\frac{\sigma}{\varkappa}$ and $k=\varkappa$.
} 
whence it follows that (2.9) is satisfied with $\gamma=\frac{1}{\tau}$.

In this paper we study a constitutive equation more general than (2.10), of the form

$$
\mathbf{q}(\mathbf{x}, t)=\int_{0}^{+\infty} K_{1}^{\prime}(s) \overline{\mathbf{g}}^{t}(\mathbf{x}, s) d s-\nabla \cdot \int_{0}^{+\infty} K_{2}^{\prime}(s) \nabla \overline{\mathbf{g}}^{t}(\mathbf{x}, s) d s,
$$

where $K_{1}$ and $K_{2} \in L^{1}\left(\mathbf{R}^{+}\right) \cap L^{2}\left(\mathbf{R}^{+}\right)$are two smooth positive functions and we have introduced the integrated history of $\mathbf{g}$, given by

$$
\overline{\mathbf{g}}^{t}(\mathbf{x}, s)=\int_{0}^{s} \mathbf{g}^{t}(\mathbf{x}, \xi) d \xi=\int_{t-s}^{t} \mathbf{g}(\mathbf{x}, \lambda) d \lambda .
$$

From (2.12), we have, after an integration by parts,

$$
\mathbf{q}(\mathbf{x}, t)=-\int_{0}^{+\infty} K_{1}(s) \mathbf{g}^{t}(\mathbf{x}, s) d s+\nabla \cdot \int_{0}^{+\infty} K_{2}(s) \nabla \mathbf{g}^{t}(\mathbf{x}, s) d s .
$$

Henceforth, since our attention shall be fixed on a specific point $\mathbf{x} \in \Omega$, the space dependence will be omitted in the following.

\section{Thermodynamics. Simple materials.}

In order to study the thermodynamic restrictions connected with the constitutive equation we firstly consider local materials 2 For these systems, the classical expression of the Second Law of Thermodynamics is given by the Clausius-Duhem inequality

$$
\dot{\eta} \geq-\nabla \cdot \frac{\mathbf{q}}{\vartheta}+\frac{r}{\vartheta}
$$

where $\vartheta$ is the absolute temperature, $\eta$ denotes the entropy and $r$ is the heat supply. Moreover, from the heat balance law we have

$$
h=-\nabla \cdot \mathbf{q}+r,
$$

where $h$ is the rate, per unit mass, at which heat is absorbed, or the internal heat power. Moreover, since we are working with a rigid conductor, the First Law takes the form

$$
\dot{e}=h,
$$

where $e$ is the internal energy. From (3.2) and (3.3), we obtain the entropy power law

$$
\frac{\dot{e}}{\vartheta}+\frac{1}{\vartheta^{2}} \mathbf{q} \cdot \nabla \vartheta=-\nabla \cdot \frac{\mathbf{q}}{\vartheta}+\frac{r}{\vartheta} \text {. }
$$

This relation, for local material, can be considered as the following balance equation,

$$
\mathcal{P}_{\eta}^{i}=\mathcal{P}_{\eta}^{e},
$$

between the internal entropy power $\mathcal{P}_{\eta}^{i}$ and the external entropy power $\mathcal{P}_{\eta}^{e}$ defined by

$$
\mathcal{P}_{\eta}^{i}=\frac{\dot{e}}{\vartheta}+\frac{1}{\vartheta^{2}} \mathbf{q} \cdot \nabla \vartheta, \quad \mathcal{P}_{\eta}^{e}=-\nabla \cdot \frac{\mathbf{q}}{\vartheta}+\frac{r}{\vartheta}
$$

Consequently, we can rewrite the inequality (3.1) as

$$
\dot{\eta} \geq \frac{\dot{e}}{\vartheta}+\frac{1}{\vartheta^{2}} \mathbf{q} \cdot \nabla \vartheta
$$

${ }^{2}$ Examples of local materials for the heat flux are given by constitutive equations such as the Fourier law (2.1) or the Cattaneo-Maxwell equation (2.7) as well as the Gurtin-Pipkin relation. 
by virtue of (3.5). Therefore, the Second Law can be represented in terms of the internal entropy power, by means of the inequality

$$
\dot{\eta} \geq \mathcal{P}_{\eta}^{i}
$$

By introducing the free energy $\psi=e-\vartheta \eta$, the inequality (3.7) can be rewritten as

$$
\dot{\psi} \leq-\eta \dot{\vartheta}-\frac{1}{\vartheta} \mathbf{q} \cdot \nabla \vartheta .
$$

Let us introduce $D(\mathbf{x}, t)$, a non-negative function referred to as the internal dissipation, which allows us to transform the inequality (3.9) into the following equality:

$$
\dot{\psi}+D=-\eta \dot{\vartheta}-\frac{1}{\vartheta} \mathbf{q} \cdot \nabla \vartheta=-\eta \dot{\vartheta}-\frac{1}{\vartheta} \mathbf{q} \cdot \mathbf{g} .
$$

Non-simple materials.

When the constitutive equation for $\mathbf{q}$ is not local, as for (2.9) and (2.12) or (2.14), we need to change the classical Clausius-Duhem inequality (3.1) or equivalently the inequality (3.7), because the internal entropy power assumes a different form. However, for non-local as well as local systems, the entropy power equation is defined by (3.4), but where the heat flux $\mathbf{q}$ is now expressed by a constitutive equation for a non-simple material. In this case, we modify the inequality (3.1) as follows:

$$
\dot{\eta} \geq-\nabla \cdot \frac{\mathbf{q}}{\vartheta}+\frac{r}{\vartheta}-\nabla \cdot \boldsymbol{\Phi}
$$

where $\mathbf{\Phi}$ denotes an extra flux which depends on the constitutive equation for q. Taking into account (3.4), this inequality assumes the form

$$
\dot{\eta} \geq \frac{\dot{e}}{\vartheta}+\frac{1}{\vartheta^{2}} \mathbf{q} \cdot \mathbf{g}-\nabla \cdot \mathbf{\Phi},
$$

which, by introducing the free energy $\psi=e-\vartheta \eta$, becomes

$$
\dot{\psi} \leq-\eta \dot{\vartheta}-\frac{1}{\vartheta} \mathbf{q} \cdot \mathbf{g}+\vartheta \nabla \cdot \mathbf{\Phi} .
$$

Following the viewpoint of [8], we consider a linearization in a neighborhood of the temperature $\vartheta_{0}$ and the null values of $\overline{\mathbf{g}}^{t}$ and $\nabla \overline{\mathbf{g}}^{t}$; therefore, in particular, we suppose that $\vartheta(x, t)=\vartheta_{0}+\epsilon \vartheta_{1}(x, t)$, where $\epsilon \ll 1$ and $\vartheta_{1}(x, t)$ is the relative temperature. Then, the linearization of (3.13) yields the following inequality:

$$
\dot{\psi} \leq-\eta \dot{\vartheta}-\frac{1}{\vartheta_{0}} \mathbf{q} \cdot \mathbf{g}+\vartheta_{0} \nabla \cdot \mathbf{\Phi}
$$

where the linearized quantities are denoted without a subscript.

4. Thermodynamic restrictions. Let $\mathcal{B}$ be a homogeneous and isotropic rigid heat conductor with memory, occupying a fixed bounded domain $\Omega \subset \mathbf{R}^{3}$ with a smooth boundary $\partial \Omega$, characterized by the constitutive equations for the heat flux given by (2.14). The memory kernels $K_{i}: \mathbf{R}^{+} \rightarrow \mathbf{R}(i=1,2)$ belong to $L^{1}\left(\mathbf{R}^{+}\right) \cap H^{1}\left(\mathbf{R}^{+}\right)$. To characterize the behaviour of such a material, we introduce a state defined by

$$
\sigma^{T}(t)=(\vartheta, \sigma(t))=\left(\vartheta, \overline{\mathbf{g}}^{t}, \nabla \overline{\mathbf{g}}^{t}\right),
$$


and a process, which is a piecewise continuous map $P^{T}:[0, d) \rightarrow \mathbf{R} \times \mathbf{R}^{3}$ defined as

$$
P^{T}(\tau)=\left(\dot{\vartheta}_{P}, P\right)=\left(\dot{\vartheta}_{P}, \mathbf{g}_{P}(\tau), \nabla \mathbf{g}_{P}(\tau)\right) \quad \forall \tau \in[0, d),
$$

where $d$, the duration of the process, generally has a finite value.

Now, we seek a free energy $\psi$ as a sum of a temperature-dependent function and a functional of the temperature gradient, specifically

$$
\psi\left(\vartheta, \overline{\mathbf{g}}^{t}, \nabla \overline{\mathbf{g}}^{t}\right)=\psi_{1}(\vartheta)+\psi_{2}\left(\overline{\mathbf{g}}^{t}, \nabla \overline{\mathbf{g}}^{t}\right) .
$$

Then, the inequality (3.14) can be satisfied if we put

$$
\begin{gathered}
\frac{\partial}{\partial \vartheta} \psi_{1}(\vartheta)=-\eta(\vartheta), \\
\dot{\psi}_{2}\left(\overline{\mathbf{g}}^{t}, \nabla \overline{\mathbf{g}}^{t}\right) \leq-\frac{1}{\vartheta_{0}} \mathbf{q} \cdot \mathbf{g}+\vartheta_{0} \nabla \cdot \boldsymbol{\Phi} .
\end{gathered}
$$

In the following we shall suppose

$$
\vartheta_{0}=1 \text {. }
$$

From the linearized constitutive equation (2.12) we have

$$
\begin{aligned}
\mathbf{q}(t) & \cdot \mathbf{g}(t)=\int_{0}^{+\infty} K_{1}^{\prime}(s) \overline{\mathbf{g}}^{t}(s) d s \cdot \mathbf{g}(t)+\int_{0}^{+\infty} K_{2}^{\prime}(s) \nabla \overline{\mathbf{g}}^{t}(s) d s \cdot \nabla \mathbf{g}(t) \\
& -\nabla \cdot\left\{\left[\int_{0}^{+\infty} K_{2}^{\prime}(s) \nabla \overline{\mathbf{g}}^{t}(s) d s\right]^{T} \mathbf{g}(t)\right\} .
\end{aligned}
$$

The transpose in the last term is irrelevant because the matrix in square brackets is symmetric. Taking

$$
\mathbf{\Phi}(t)=-\left[\int_{0}^{+\infty} K_{2}^{\prime}(s) \nabla \overline{\mathbf{g}}^{t}(s) d s\right] \mathbf{g}(t),
$$

the inequality (4.5), by virtue of (4.6), reduces to

$$
\begin{aligned}
\dot{\psi}_{2}(t) & \leq-\mathbf{q} \cdot \mathbf{g}+\nabla \cdot \mathbf{\Phi} \\
& \leq-\int_{0}^{+\infty} K_{1}^{\prime}(s) \overline{\mathbf{g}}^{t}(s) d s \cdot \mathbf{g}(t)-\int_{0}^{+\infty} K_{2}^{\prime}(s) \nabla \overline{\mathbf{g}}^{t}(s) d s \cdot \nabla \mathbf{g}(t) .
\end{aligned}
$$

By introducing the entropy action

$$
\begin{gathered}
\mathcal{A}(t)=\mathcal{A}(\sigma, P)=-\int_{0}^{+\infty} K_{1}^{\prime}(s) \overline{\mathbf{g}}^{t}(s) d s \cdot \mathbf{g}(t) \\
-\int_{0}^{+\infty} K_{2}^{\prime}(s) \nabla \overline{\mathbf{g}}^{t}(s) d s \cdot \nabla \mathbf{g}(t),
\end{gathered}
$$

the inequality $(4.9)_{2}$ can be rewritten as

$$
\dot{\psi}_{2}\left(\overline{\mathbf{g}}^{t}, \nabla \overline{\mathbf{g}}^{t}\right) \leq \mathcal{A}(t),
$$

with which, as for (3.10), we can associate an internal dissipation $D_{2}(\mathbf{x}, t)$, a non-negative function obeying

$$
\dot{\psi}_{2}(t)+D_{2}(t)=\mathcal{A}(t)
$$


The inequality (4.9) 1 is equivalent to the representation (4.11) by virtue of an extra flux $\boldsymbol{\Phi}(t)$, which for a local material is zero, while for non-local systems depends on the material (see I. Müller [20]). Using (4.7) and (4.10), we have

$$
\mathcal{A}(t)=-\mathbf{q}(t) \cdot \mathbf{g}(t)+\nabla \cdot \mathbf{\Phi}(t),
$$

where $\boldsymbol{\Phi}(t)$ is given by (4.8). We can write (4.10) in the form

$$
\begin{aligned}
\mathcal{A}(t)=\int_{0}^{+\infty} K_{1}(s) \mathbf{g}^{t}(s) d s \cdot \mathbf{g}(t) \\
\quad+\int_{0}^{+\infty} K_{2}(s) \nabla \mathbf{g}^{t}(s) d s \cdot \nabla \mathbf{g}(t),
\end{aligned}
$$

with the aid of partial integrations. Extending the integration to $\mathbf{R}$ but defining $\mathbf{g}^{t}(s)$, $\nabla \mathbf{g}^{t}(s), s<0$, to be zero, we have the alternative form:

$$
\begin{aligned}
\mathcal{A}(t)= & \int_{-\infty}^{+\infty} K_{1}(|s|) \mathbf{g}^{t}(s) d s \cdot \mathbf{g}(t) \\
& +\int_{-\infty}^{+\infty} K_{2}(|s|) \nabla \mathbf{g}^{t}(s) d s \cdot \nabla \mathbf{g}(t) .
\end{aligned}
$$

The quantity $\mathcal{A}(t)$ allows us to evaluate the total entropy action $\mathcal{B}(\sigma, P)$ of the material during the application of a process $P$ of duration $d$,

$$
\mathcal{B}(\sigma, P)=\int_{t}^{t+d} \mathcal{A}(\xi) d \xi
$$

We now assert the following principle, which is a consequence of the Second Law of Thermodynamics, as expressed by (4.12).

Dissipation principle. On any cycle $(\sigma, P)$ we have

$$
\mathcal{B}(\sigma, P) \geq 0,
$$

where the equality sign holds if and only if the cycle is reversible.

Using (2.14) instead of (2.12) in (4.7), we obtain an alternative form for $\mathcal{B}(\sigma, P)$, given by

$$
\begin{aligned}
\mathcal{B}(\sigma, P)=\int_{t}^{t+d}[ & \int_{0}^{+\infty} K_{1}(s) \mathbf{g}^{\tau}(s) d s \cdot \mathbf{g}(\tau) \\
& \left.+\int_{0}^{+\infty} K_{2}(s) \nabla \mathbf{g}^{\tau}(s) d s \cdot \nabla \mathbf{g}(\tau)\right] d \tau .
\end{aligned}
$$

This expression shall be used to obtain the thermodynamic restrictions imposed on the constitutive equation (2.14).

Let us assume periodic histories for $\mathbf{g}$ and its gradient $\nabla \mathbf{g}$ of the form

$$
\mathbf{g}(s)=\cos \omega s \mathbf{a}_{1}+\sin \omega s \mathbf{a}_{2}, \nabla \mathbf{g}(s)=\cos \omega s \mathbf{A}_{1}+\sin \omega s \mathbf{A}_{2}
$$

for any $s \leq t$ and $\omega \in \mathbb{R} \backslash\{0\}$, where $\mathbf{a}_{i}, \mathbf{A}_{i}(i=1,2)$ denote arbitrary non-zero vectors and second-order tensors depending only of $\mathbf{x}$, while $d=2 \pi /|\omega|$ is the period of the 
history. Substituting (4.19) into (4.18), and invoking periodicity to change the interval of integration $(t, t+d)$ to $(0, d)$, we obtain

$$
\begin{aligned}
\mathcal{B}(\sigma, P)=\int_{0}^{d} \quad\left\{\int_{0}^{+\infty} K_{1}(s)\left[\cos \omega(t-s) \mathbf{a}_{1}+\sin \omega(t-s) \mathbf{a}_{2}\right] d s\right. \\
\cdot\left[\cos \omega t \mathbf{a}_{1}+\sin \omega t \mathbf{a}_{2}\right]+\int_{0}^{+\infty} K_{2}(s)\left[\cos \omega(t-s) \mathbf{A}_{1}\right. \\
\left.\left.+\sin \omega(t-s) \mathbf{A}_{2}\right] d s \cdot\left[\cos \omega t \mathbf{A}_{1}+\sin \omega t \mathbf{A}_{2}\right]\right\} d t>0
\end{aligned}
$$

where $\tau$ has been replaced by $t$. Integrating with respect to $t$, we find that

$$
\mathcal{B}(\sigma, P)=\frac{d}{2}\left[\left(\left|\mathbf{a}_{1}\right|^{2}+\left|\mathbf{a}_{2}\right|^{2}\right) K_{1_{c}}(\omega)+\left(\left|\mathbf{A}_{1}\right|^{2}+\left|\mathbf{A}_{2}\right|^{2}\right) K_{2_{c}}(\omega)\right]>0,
$$

where $K_{1_{c}}(\omega)$ and $K_{2_{c}}(\omega)$ are the half-range Fourier cosine transforms of the kernels $K_{1}(s)$ and $K_{2}(s)$, given by $(17.3)$. Also (no summation over repeated indices)

$$
\left|\mathbf{a}_{i}\right|^{2}=\mathbf{a}_{i} \cdot \mathbf{a}_{i} \quad(i=1,2)
$$

and

$$
\left|\mathbf{A}_{i}\right|^{2}=\operatorname{Tr}\left(\mathbf{A}_{i} \mathbf{A}_{i}^{\top}\right) \quad(i=1,2) .
$$

It follows from the arbitrariness of $\mathbf{a}_{i}, \mathbf{A}_{i}(i=1,2)$ that

$$
K_{1_{c}}(\omega)>0, \quad K_{2_{c}}(\omega)>0 \quad \forall \omega \in \mathbb{R} \backslash\{0\} .
$$

Under the hypothesis that

$$
\begin{aligned}
& K_{1_{c}}(0)=\int_{0}^{+\infty} K_{1}(s) d s:=\nu_{1_{\infty}} \neq 0, \\
& K_{2_{c}}(0)=\int_{0}^{+\infty} K_{1}(s) d s:=\nu_{2_{\infty}} \neq 0,
\end{aligned}
$$

we can extend (4.24) to $\mathbb{R}$.

The inequality (4.25) is also a sufficient condition for the compatibility of the constitutive equation with the Dissipation Principle; this proof is analogous to that given in [11.

We also note that, under the hypotheses assumed for $K_{i}(i=1,2)$, using the inverse half-range Fourier transform, (4.24) and (7.5), we have

$$
\begin{aligned}
& K_{i}(t)=\frac{2}{\pi} \int_{0}^{+\infty} K_{i_{c}}(\omega) \cos \omega t d \omega \\
& K_{i}(0)=\frac{2}{\pi} \int_{0}^{+\infty} K_{i_{c}}(\omega) d \omega>0(i=1,2) .
\end{aligned}
$$

Also, from (7.6),

$$
\lim _{\omega \rightarrow+\infty} \omega K_{i_{s}}^{\prime}(\omega)=-\lim _{\omega \rightarrow+\infty} \omega^{2} K_{i_{c}}(\omega)=K_{i}^{\prime}(0) \leq 0 \quad(i=1,2) .
$$

It will be assumed that

$$
K_{i}^{\prime}(0)<0 \quad(i=1,2)
$$


For constant histories

$$
\mathbf{g}^{t}(s)=\mathbf{g}, \quad \nabla \mathbf{g}^{t}(s)=\nabla \mathbf{g} \quad \forall s \in \mathbf{R}^{+},
$$

we obtain from (2.14) the following heat flux,

$$
\mathbf{q}(t)=-\nu_{1_{\infty}} \mathbf{g}+\nu_{2_{\infty}} \nabla \cdot \nabla \mathbf{g}
$$

with the aid of (4.25).

5. Free energies for the rigid heat conductor. We now consider two candidate expressions for the free energy $\psi_{2}\left(\overline{\mathbf{g}}^{t}, \nabla \overline{\mathbf{g}}^{t}\right)$ introduced in (4.3), based on functionals which have already been considered for linear viscoelastic solids and rigid heat conductors [1, 2] in the case of simple materials. Our aim here is to generalize these functionals to non-simple materials.

5.1. The Graff-Volterra free energy. An important functional, frequently used in applications for viscoelastic solids, is the Graffi-Volterra free energy [16, 17, 22. Such a functional can be generalized to our non-simple heat conductors with the following form:

$$
\begin{aligned}
\psi_{\mathcal{G}}(t)=- & \frac{1}{2} \int_{0}^{+\infty} K_{1}^{\prime}(s) \overline{\mathbf{g}}^{t}(s) \cdot \overline{\mathbf{g}}^{t}(s) d s \\
& -\frac{1}{2} \int_{0}^{+\infty} K_{2}^{\prime}(s) \nabla \overline{\mathbf{g}}^{t}(s) \cdot \nabla \overline{\mathbf{g}}^{t}(s) d s,
\end{aligned}
$$

which is non-negative if we assume that

$$
K_{i}^{\prime}(s)<0 \forall s \in \mathbb{R}^{+}(i=1,2) .
$$

Another constraint will be imposed on these kernel functions to ensure a non-negative rate of dissipation, namely

$$
K_{i}^{\prime \prime}(s) \geq 0 \forall s \in \mathbb{R}^{+}(i=1,2) .
$$

Taking account of

$$
\frac{d}{d t} \overline{\mathbf{g}}^{t}(s)=\mathbf{g}(t)-\frac{d}{d s} \overline{\mathbf{g}}^{t}(s),
$$

and the analogous relation for $\frac{d}{d t} \nabla \overline{\mathbf{g}}^{t}(s)$, the derivative with respect to time of (5.1) gives, with the aid of an integration by parts,

$$
\begin{aligned}
\dot{\psi}_{\mathcal{G}}(t)=- & \int_{0}^{+\infty} K_{1}^{\prime}(s) \overline{\mathbf{g}}^{t}(s) \cdot \frac{d}{d t} \overline{\mathbf{g}}^{t}(s) d s \\
& -\int_{0}^{+\infty} K_{2}^{\prime}(s) \nabla \overline{\mathbf{g}}^{t}(s) \cdot \frac{d}{d t} \nabla \overline{\mathbf{g}}^{t}(s) d s \\
= & -\int_{0}^{+\infty} K_{1}^{\prime}(s) \overline{\mathbf{g}}^{t}(s) d s \cdot \mathbf{g}(t)-\int_{0}^{+\infty} K_{2}^{\prime}(s) \nabla \overline{\mathbf{g}}^{t}(s) d s \cdot \nabla \mathbf{g}(t) \\
& -\frac{1}{2} \int_{0}^{+\infty} K_{1}^{\prime \prime}(s)\left[\overline{\mathbf{g}}^{t}(s)\right]^{2} d s-\frac{1}{2} \int_{0}^{+\infty} K_{2}^{\prime \prime}(s)\left[\nabla \overline{\mathbf{g}}^{t}(s)\right]^{2} d s \\
= & \mathcal{A}(t)-D_{\mathcal{G}}(t),
\end{aligned}
$$


which satisfies (4.12), by virtue of (4.10), provided we take

$$
D_{\mathcal{G}}(t)=\frac{1}{2} \int_{0}^{+\infty} K_{1}^{\prime \prime}(s)\left[\overline{\mathbf{g}}^{t}(s)\right]^{2} d s+\frac{1}{2} \int_{0}^{+\infty} K_{2}^{\prime \prime}(s)\left[\nabla \overline{\mathbf{g}}^{t}(s)\right]^{2} d s \geq 0 .
$$

This is the non-negative internal dissipation corresponding to the Graffi-Volterra functional.

We can derive from $(5.5)_{2}$ the relation $(4.9)_{1}$ (or (4.12) with (4.13) $)$ in terms of the extra flux, since we have

$$
\begin{aligned}
\dot{\psi}_{\mathcal{G}}(t)=- & {\left[\int_{0}^{+\infty} K_{1}^{\prime}(s) \overline{\mathbf{g}}^{t}(s) d s-\nabla \cdot \int_{0}^{+\infty} K_{2}^{\prime}(s) \nabla \overline{\mathbf{g}}^{t}(s) d s\right] \cdot \mathbf{g}(t) } \\
- & \frac{1}{2} \int_{0}^{+\infty} K_{1}^{\prime \prime}(s)\left[\overline{\mathbf{g}}^{t}(s)\right]^{2} d s-\frac{1}{2} \int_{0}^{+\infty} K_{2}^{\prime \prime}(s)\left[\nabla \overline{\mathbf{g}}^{t}(s)\right]^{2} d s \\
& +\nabla \cdot\left\{\left[-\int_{0}^{+\infty} K_{2}^{\prime}(s) \nabla \overline{\mathbf{g}}^{t}(s) d s\right] \mathbf{g}(t)\right\} \\
=- & \mathbf{q}(t) \cdot \mathbf{g}(t)-D_{\mathcal{G}}(t)+\nabla \cdot \mathbf{\Phi}(t) \leq-\mathbf{q}(t) \cdot \mathbf{g}(t)+\nabla \cdot \mathbf{\Phi}(t),
\end{aligned}
$$

where (4.8) has been invoked.

5.2. A new free energy in terms of the minimal state. We now consider the new free energy $\psi_{F}$, recently introduced and considered, in particular, in [7] and [6] for viscoelastic solids. For this purpose let us introduce the quantities

$$
\begin{aligned}
\mathbf{I}^{t}\left(\tau, \overline{\mathbf{g}}^{t}\right) & =\int_{0}^{+\infty} K_{1}^{\prime}(\tau+\eta) \overline{\mathbf{g}}^{t}(\eta) d \eta, \\
\mathfrak{I}^{t}\left(\tau, \nabla \overline{\mathbf{g}}^{t}\right) & =\int_{0}^{+\infty} K_{2}^{\prime}(\tau+\eta) \nabla \overline{\mathbf{g}}^{t}(\eta) d \eta,
\end{aligned}
$$

which define a minimal state. In particular, we have

$$
\mathbf{I}^{t}\left(0, \overline{\mathbf{g}}^{t}\right)=\int_{0}^{+\infty} K_{1}^{\prime}(\eta) \overline{\mathbf{g}}^{t}(\eta) d \eta, \mathfrak{I}^{t}\left(0, \nabla \overline{\mathbf{g}}^{t}\right)=\int_{0}^{+\infty} K_{2}^{\prime}(\eta) \nabla \overline{\mathbf{g}}^{t}(\eta) d \eta .
$$

Moreover, their derivatives with respect to $\tau$ are given by

$$
\begin{aligned}
\mathbf{I}_{(1)}^{t}\left(\tau, \overline{\mathbf{g}}^{t}\right) & =\int_{0}^{+\infty} K_{1}^{\prime \prime}(\tau+\eta) \overline{\mathbf{g}}^{t}(\eta) d \eta, \\
\mathfrak{I}_{(1)}^{t}\left(\tau, \nabla \overline{\mathbf{g}}^{t}\right) & =\int_{0}^{+\infty} K_{2}^{\prime \prime}(\tau+\eta) \nabla \overline{\mathbf{g}}^{t}(\eta) d \eta,
\end{aligned}
$$

which yield

$$
\begin{aligned}
\mathbf{I}_{(1)}^{t}\left(0, \overline{\mathbf{g}}^{t}\right) & =\int_{0}^{+\infty} K_{1}^{\prime \prime}(\eta) \overline{\mathbf{g}}^{t}(\eta) d \eta, \\
\mathfrak{I}_{(1)}^{t}\left(0, \nabla \overline{\mathbf{g}}^{t}\right) & =\int_{0}^{+\infty} K_{2}^{\prime \prime}(\eta) \nabla \overline{\mathbf{g}}^{t}(\eta) d \eta .
\end{aligned}
$$


Now, consider the following functional:

$$
\begin{aligned}
\psi_{\mathcal{F}}^{(\mathbf{q})}(t)= & -\frac{1}{2} \int_{0}^{+\infty} \frac{1}{K_{1}^{\prime}(\tau)}\left[\mathbf{I}_{(1)}^{t}\left(\tau, \overline{\mathbf{g}}^{t}\right)\right]^{2} d \tau \\
& -\frac{1}{2} \int_{0}^{+\infty} \frac{1}{K_{2}^{\prime}(\tau)}\left[\mathfrak{I}_{(1)}^{t}\left(\tau, \nabla \overline{\mathbf{g}}^{t}\right)\right]^{2} d \tau
\end{aligned}
$$

under the hypotheses that $K_{i}^{\prime}(s)$ and $K_{i}^{\prime \prime}(s)(i=1,2)$ satisfy (5.2) and (5.3). In order to verify that this expression gives a possible free energy $\psi_{2}\left(\overline{\mathbf{g}}^{t}, \nabla \overline{\mathbf{g}}^{t}\right)$ for our body, we evaluate its derivative with respect to $t$, which takes the form

$$
\begin{aligned}
\dot{\psi}_{\mathcal{F}}^{(\mathbf{q})}(t)= & -\int_{0}^{+\infty} \frac{1}{K_{1}^{\prime}(\tau)} \dot{\mathbf{I}}_{(1)}^{t}\left(\tau, \overline{\mathbf{g}}^{t}\right) \cdot \mathbf{I}_{(1)}^{t}\left(\tau, \overline{\mathbf{g}}^{t}\right) d \tau \\
& -\int_{0}^{+\infty} \frac{1}{K_{2}^{\prime}(\tau)} \dot{\mathfrak{I}}_{(1)}^{t}\left(\tau, \nabla \overline{\mathbf{g}}^{t}\right) \cdot \mathfrak{I}_{(1)}^{t}\left(\tau, \nabla \overline{\mathbf{g}}^{t}\right) d \tau .
\end{aligned}
$$

Then, from (5.10), by using (5.4) and integrating by parts, we obtain

$$
\begin{gathered}
\dot{\mathbf{I}}_{(1)}^{t}\left(\tau, \overline{\mathbf{g}}^{t}\right) \equiv \frac{d}{d t} \mathbf{I}_{(1)}^{t}\left(\tau, \overline{\mathbf{g}}^{t}\right)=-K_{1}^{\prime}(\tau) \mathbf{g}(t)+\mathbf{I}_{(2)}^{t}\left(\tau, \overline{\mathbf{g}}^{t}\right), \\
\dot{\mathfrak{I}}_{(1)}^{t}\left(\tau, \nabla \overline{\mathbf{g}}^{t}\right) \equiv \frac{d}{d t} \Im_{(1)}^{t}\left(\tau, \nabla \overline{\mathbf{g}}^{t}\right)=-K_{2}^{\prime}(\tau) \nabla \mathbf{g}(t)+\mathfrak{I}_{(2)}^{t}\left(\tau, \nabla \overline{\mathbf{g}}^{t}\right),
\end{gathered}
$$

with

$$
\mathbf{I}_{(2)}^{t}\left(\tau, \overline{\mathbf{g}}^{t}\right) \equiv \frac{d}{d \tau} \mathbf{I}_{(1)}^{t}\left(\tau, \overline{\mathbf{g}}^{t}\right), \quad \mathfrak{I}_{(2)}^{t}\left(\tau, \nabla \overline{\mathbf{g}}^{t}\right) \equiv \frac{d}{d \tau} \mathfrak{I}_{(1)}^{t}\left(\tau, \nabla \overline{\mathbf{g}}^{t}\right)
$$

Then, by substituting (5.14) and (5.15) into (5.13), we have

$$
\begin{aligned}
\dot{\psi}_{\mathcal{F}}^{(\mathbf{q})}(t)=- & \mathbf{I}^{t}\left(0, \overline{\mathbf{g}}^{t}\right) \cdot \mathbf{g}(t)-\mathfrak{I}^{t}\left(0, \nabla \overline{\mathbf{g}}^{t}\right) \cdot \nabla \mathbf{g}(t) \\
& -\frac{1}{2} \int_{0}^{+\infty} \frac{K_{1}^{\prime \prime}(\tau)}{\left[K_{1}^{\prime}(\tau)\right]^{2}}\left[\mathbf{I}_{(1)}^{t}\left(\tau, \overline{\mathbf{g}}^{t}\right)\right]^{2} d \tau \\
& -\frac{1}{2} \int_{0}^{+\infty} \frac{K_{2}^{\prime \prime}(\tau)}{\left[K_{2}^{\prime}(\tau)\right]^{2}}\left[\mathfrak{I}_{(1)}^{t}\left(\tau, \nabla \overline{\mathbf{g}}^{t}\right)\right]^{2} d \tau \\
& +\frac{1}{2} \frac{1}{K_{1}^{\prime}(0)}\left[\mathbf{I}_{(1)}^{t}\left(0, \overline{\mathbf{g}}^{t}\right)\right]^{2}+\frac{1}{2} \frac{1}{K_{2}^{\prime}(0)}\left[\mathfrak{I}_{(1)}^{t}\left(0, \nabla \overline{\mathbf{g}}^{t}\right)\right]^{2},
\end{aligned}
$$


whence, using (5.9), it follows that

$$
\begin{aligned}
\dot{\psi}_{\mathcal{F}}^{(\mathbf{q})}(t)= & -\left[\int_{0}^{+\infty} K_{1}^{\prime}(\eta) \overline{\mathbf{g}}^{t}(\eta) d \eta \cdot \mathbf{g}=(t)\right. \\
& \left.+\int_{0}^{+\infty} K_{2}^{\prime}(\eta) \nabla \overline{\mathbf{g}}^{t}(\eta) d \eta \cdot \nabla \mathbf{g}(t)\right] \\
& -\frac{1}{2} \int_{0}^{+\infty} \frac{K_{1}^{\prime \prime}(\tau)}{\left[K_{1}^{\prime}(\tau)\right]^{2}}\left[\mathbf{I}_{(1)}^{t}\left(\tau, \overline{\mathbf{g}}^{t}\right)\right]^{2} d \tau \\
& -\frac{1}{2} \int_{0}^{+\infty} \frac{K_{2}^{\prime \prime}(\tau)}{\left[K_{2}^{\prime}(\tau)\right]^{2}}\left[\mathfrak{I}_{(1)}^{t}\left(\tau, \nabla \overline{\mathbf{g}}^{t}\right)\right]^{2} d \tau \\
& +\frac{1}{2} \frac{1}{K_{1}^{\prime}(0)}\left[\mathbf{I}_{(1)}^{t}\left(0, \overline{\mathbf{g}}^{t}\right)\right]^{2}+\frac{1}{2} \frac{1}{K_{2}^{\prime}(0)}\left[\mathfrak{I}_{(1)}^{t}\left(0, \nabla \overline{\mathbf{g}}^{t}\right)\right]^{2} \\
= & \mathcal{A}(t)-D_{\mathcal{G}}(t) \leq \mathcal{A}(t),
\end{aligned}
$$

by virtue of (4.10); hence, the equality (4.12) is satisfied with the following form for the internal dissipation:

$$
\begin{aligned}
D_{\mathcal{F}}(t)= & \frac{1}{2} \int_{0}^{+\infty} \frac{K_{1}^{\prime \prime}(\tau)}{\left[K_{1}^{\prime}(\tau)\right]^{2}}\left[\mathbf{I}_{(1)}^{t}\left(\tau, \overline{\mathbf{g}}^{t}\right)\right]^{2} d \tau \\
& +\frac{1}{2} \int_{0}^{+\infty} \frac{K_{2}^{\prime \prime}(\tau)}{\left[K_{2}^{\prime}(\tau)\right]^{2}}\left[\mathfrak{I}_{(1)}^{t}\left(\tau, \nabla \overline{\mathbf{g}}^{t}\right)\right]^{2} d \tau \\
& -\frac{1}{2} \frac{1}{K_{1}^{\prime}(0)}\left[\mathbf{I}_{(1)}^{t}\left(0, \overline{\mathbf{g}}^{t}\right)\right]^{2}-\frac{1}{2} \frac{1}{K_{2}^{\prime}(0)}\left[\mathfrak{I}_{(1)}^{t}\left(0, \nabla \overline{\mathbf{g}}^{t}\right)\right]^{2} \geq 0,
\end{aligned}
$$

because of the hypotheses (5.2) and (5.3) for $K_{i}^{\prime}(s)$ and $K_{i}^{\prime \prime}(s)(i=1,2)$. As in (5.7), the relation (5.18) 1 can also be written in the form

$$
\begin{aligned}
\dot{\psi}_{\mathcal{F}}^{(\mathbf{q})}(t)= & -\left[\int_{0}^{+\infty} K_{1}^{\prime}(\eta) \overline{\mathbf{g}}^{t}(\eta) d \eta-\nabla \cdot \int_{0}^{+\infty} K_{2}^{\prime}(\eta) \nabla \overline{\mathbf{g}}^{t}(\eta) d \eta\right] \cdot \mathbf{g}(t) \\
& +\nabla \cdot\left\{-\left[\int_{0}^{+\infty} K_{2}^{\prime}(\eta) \nabla \overline{\mathbf{g}}^{t}(\eta) d \eta\right] \mathbf{g}(t)\right\} \\
& -\frac{1}{2} \int_{0}^{+\infty} \frac{K_{1}^{\prime \prime}(\tau)}{\left[K_{1}^{\prime}(\tau)\right]^{2}}\left[\mathbf{I}_{(1)}^{t}\left(\tau, \overline{\mathbf{g}}^{t}\right)\right]^{2} d \tau \\
& -\frac{1}{2} \int_{0}^{+\infty} \frac{K_{2}^{\prime \prime}(\tau)}{\left[K_{2}^{\prime}(\tau)\right]^{2}}\left[\mathfrak{I}_{(1)}^{t}\left(\tau, \nabla \overline{\mathbf{g}}^{t}\right)\right]^{2} d \tau \\
& +\frac{1}{2} \frac{1}{K_{1}^{\prime}(0)}\left[\mathbf{I}_{(1)}^{t}\left(0, \overline{\mathbf{g}}^{t}\right)\right]^{2}+\frac{1}{2} \frac{1}{K_{2}^{\prime}(0)}\left[\mathfrak{I}_{(1)}^{t}\left(0, \nabla \overline{\mathbf{g}}^{t}\right)\right]^{2} \\
= & -\mathbf{q}(t) \cdot \mathbf{g}(t)-D_{\mathcal{G}}(t)+\nabla \cdot \mathbf{\Phi}(t) \leq-\mathbf{q}(t) \cdot \mathbf{g}(t)+\nabla \cdot \mathbf{\Phi}(t)
\end{aligned}
$$

which is $(4.9) 1$ with $\boldsymbol{\Phi}$ expressed by (4.8). 
6. The minimum free energy. Applying partial integrations, (5.8) can also be written in the form

$$
\begin{aligned}
\mathbf{I}^{t}\left(\tau, \overline{\mathbf{g}}^{t}\right) & =-\int_{0}^{+\infty} K_{1}(\tau+\eta) \mathbf{g}^{t}(\eta) d \eta \\
\mathfrak{I}^{t}\left(\tau, \nabla \overline{\mathbf{g}}^{t}\right) & =-\int_{0}^{+\infty} K_{2}(\tau+\eta) \nabla \mathbf{g}^{t}(\eta) d \eta .
\end{aligned}
$$

We can write these as

$$
\begin{aligned}
\mathbf{I}^{t}\left(\tau, \overline{\mathbf{g}}^{t}\right) & =-\int_{-\infty}^{0} K_{1}(\tau-\eta) \mathbf{g}^{t}(-\eta) d \eta \\
& =-\int_{-\infty}^{0} K_{1}(|\tau-\eta|) \mathbf{g}^{t}(-\eta) d \eta, \\
\mathfrak{I}^{t}\left(\tau, \nabla \overline{\mathbf{g}}^{t}\right) & =-\int_{-\infty}^{0} K_{2}(\tau-\eta) \nabla \mathbf{g}^{t}(-\eta) d \eta \\
& =-\int_{-\infty}^{0} K_{2}(|\tau-\eta|) \nabla \mathbf{g}^{t}(-\eta) d \eta .
\end{aligned}
$$

Let us now consider the total entropy action $\mathcal{B}(\sigma, P)$ of the material during the application of a process $P(\tau)=\left(\mathbf{g}_{P}(\tau), \nabla \mathbf{g}_{P}(\tau)\right) \forall \tau \in[0, d)$, starting at time $t$ when the state is $\sigma(t)=\left(\overline{\mathbf{g}}^{t}, \nabla \overline{\mathbf{g}}^{t}\right)$. Its expression is given by (4.16), with (4.10), which can be rewritten as

$$
\begin{aligned}
\mathcal{B}(\sigma, P) & =\int_{0}^{d}\left[-\mathbf{q}(t+\tau) \cdot \mathbf{g}_{P}(\tau)+\nabla \cdot \mathbf{\Phi}(t+\tau)\right] d \tau, \\
\mathbf{g}_{P}(\tau) & =\mathbf{g}(t+\tau)
\end{aligned}
$$

with the aid of (4.13). Here we must consider the restriction $P_{\tau}$ of the process and the related continuations of the initial histories, expressed by

$$
\begin{aligned}
\overline{\mathbf{g}}^{t+\tau}(s) & =\left(\overline{\mathbf{g}}_{P} * \overline{\mathbf{g}}\right)^{t+\tau}(s) \\
& = \begin{cases}\int_{\tau-s}^{\tau} \mathbf{g}_{P}(s) d s=\overline{\mathbf{g}}_{P}^{\tau}(s) & \forall s \in[0, \tau), \\
\overline{\mathbf{g}}_{P}^{\tau}(\tau)+\overline{\mathbf{g}}^{t}(s-\tau) & \forall s \geq \tau,\end{cases} \\
\nabla \overline{\mathbf{g}}^{t+\tau}(s) & =\left(\nabla \overline{\mathbf{g}}_{P} * \nabla \overline{\mathbf{g}}\right)^{t+\tau}(s) \\
& = \begin{cases}\int_{\tau-s}^{\tau} \nabla \mathbf{g}_{P}(s) d s=\nabla \overline{\mathbf{g}}_{P}^{\tau}(s) & \forall s \in[0, \tau), \\
\nabla \overline{\mathbf{g}}_{P}^{\tau}(\tau)+\nabla \overline{\mathbf{g}}^{t}(s-\tau) & \forall s \geq \tau,\end{cases}
\end{aligned}
$$

which, with some integrations, allow us to obtain from (2.12),

$$
\begin{aligned}
\mathbf{q}(t+\tau)= & \int_{0}^{\tau} K_{1}^{\prime}(s) \overline{\mathbf{g}}_{P}^{\tau}(s) d s+\int_{\tau}^{+\infty} K_{1}^{\prime}(s)\left[\overline{\mathbf{g}}_{P}^{\tau}(\tau)+\overline{\mathbf{g}}^{t}(s-\tau)\right] d s \\
& -\nabla \cdot\left\{\int_{0}^{\tau} K_{2}^{\prime}(s) \nabla \overline{\mathbf{g}}_{P}^{\tau}(s) d s+\int_{\tau}^{+\infty} K_{2}^{\prime}(s)\left[\nabla \overline{\mathbf{g}}_{P}^{\tau}(\tau)+\nabla \overline{\mathbf{g}}^{t}(s-\tau)\right] d s\right\} \\
= & -\int_{0}^{\tau} K_{1}(s) \mathbf{g}_{P}^{\tau}(s) d s+\int_{0}^{+\infty} K_{1}^{\prime}(\xi+\tau) \overline{\mathbf{g}}^{t}(\xi) d \xi \\
& +\nabla \cdot \int_{0}^{\tau} K_{2}(s) \nabla \mathbf{g}_{P}^{\tau}(s) d s-\nabla \cdot \int_{0}^{+\infty} K_{2}^{\prime}(\xi+\tau) \nabla \overline{\mathbf{g}}^{t}(\xi) d \xi
\end{aligned}
$$


and from (4.8),

$$
\begin{aligned}
\nabla \cdot \mathbf{\Phi}(t+\tau)= & -\nabla \cdot\left\{\int_{0}^{\tau} K_{2}^{\prime}(s) \nabla \overline{\mathbf{g}}_{P}^{\tau}(s) d s+\int_{\tau}^{+\infty} K_{2}^{\prime}(s)\left[\nabla \overline{\mathbf{g}}_{P}^{\tau}(\tau)\right.\right. \\
& \left.\left.+\nabla \overline{\mathbf{g}}^{t}(s-\tau)\right] d s\right\} \cdot \mathbf{g}_{P}(\tau)-\left\{\int_{0}^{\tau} K_{2}^{\prime}(s) \nabla \overline{\mathbf{g}}_{P}^{\tau}(s) d s\right. \\
& \left.+\int_{\tau}^{+\infty} K_{2}^{\prime}(\tau)\left[\nabla \overline{\mathbf{g}}_{P}^{\tau}(\tau)+\nabla \overline{\mathbf{g}}^{t}(s-\tau)\right] d s\right\} \cdot \nabla \mathbf{g}_{P}(\tau) \\
= & \left\{\nabla \cdot \int_{0}^{\tau} K_{2}(s) \nabla \mathbf{g}_{P}^{\tau}(s) d s-\nabla \cdot \int_{0}^{+\infty} K_{2}^{\prime}(\xi+\tau) \nabla \overline{\mathbf{g}}^{t}(\xi) d \xi\right\} \cdot \mathbf{g}_{P}(\tau) \\
& +\left\{\int_{0}^{\tau} K_{2}(s) \nabla \mathbf{g}_{P}^{\tau}(s) d s-\int_{0}^{+\infty} K_{2}^{\prime}(\xi+\tau) \nabla \overline{\mathbf{g}}^{t}(\xi) d \xi\right\} \cdot \nabla \overline{\mathbf{g}}_{P}(\tau) .
\end{aligned}
$$

Substituting (6.6) and (6.7) into (6.3), we obtain

$$
\begin{aligned}
& \mathcal{B}(\sigma, P)=\int_{0}^{d}\left\{\left[\int_{0}^{\tau} K_{1}(s) \mathbf{g}_{P}^{\tau}(s) d s-\int_{0}^{+\infty} K_{1}^{\prime}(\xi+\tau) \overline{\mathbf{g}}^{t}(\xi) d \xi\right] \cdot \mathbf{g}_{P}(\tau)\right. \\
& \left.+\left[\int_{0}^{\tau} K_{2}(s) \nabla \mathbf{g}_{P}^{\tau}(s) d s-\int_{0}^{+\infty} K_{2}^{\prime}(\xi+\tau) \nabla \overline{\mathbf{g}}^{t}(\xi) d \xi\right] \cdot \nabla \mathbf{g}_{P}(\tau)\right\} d \tau .
\end{aligned}
$$

Let us extend the process on $\mathbf{R}^{+}$by means of $P(\tau)=(0, \mathbf{0}), \forall \tau>d$; thus, (6.8) becomes

$$
\begin{aligned}
\mathcal{B}(\sigma, P)= & \int_{0}^{+\infty}\left\{\left[\int_{0}^{\tau} K_{1}(\tau-\eta) \mathbf{g}_{P}(\eta) d \eta-\mathbf{I}^{t}\left(\tau, \overline{\mathbf{g}}^{t}\right)\right] \cdot \mathbf{g}_{P}(\tau)\right. \\
& \left.+\left[\int_{0}^{\tau} K_{2}(\tau-\eta) \nabla \mathbf{g}_{P}(\eta) d \eta-\mathfrak{I}^{t}\left(\tau, \nabla \overline{\mathbf{g}}^{t}\right)\right] \cdot \nabla \mathbf{g}_{P}(\tau)\right\} d \tau \\
= & \int_{0}^{+\infty}\left[\frac{1}{2} \int_{0}^{+\infty} K_{1}(|\tau-\eta|) \mathbf{g}_{P}(\eta) d \eta-\mathbf{I}^{t}\left(\tau, \overline{\mathbf{g}}^{t}\right)\right] \cdot \mathbf{g}_{P}(\tau) d \tau \\
& +\int_{0}^{+\infty}\left[\frac{1}{2} \int_{0}^{+\infty} K_{2}(|\tau-\eta|) \nabla \mathbf{g}_{P}(\eta) d \eta-\mathfrak{I}^{t}\left(\tau, \nabla \overline{\mathbf{g}}^{t}\right)\right] \cdot \nabla \mathbf{g}_{P}(\tau) d \tau
\end{aligned}
$$

The minimum free energy $\psi_{m}(t)$ coincides with the maximum recoverable action $\mathcal{B}_{R}(\sigma)$,

$$
\psi_{m}(t) \equiv \mathcal{B}_{R}(\sigma)=\sup \{-\mathcal{B}(\sigma, P): P \in \Pi\},
$$

which is the maximum quantity of action we can obtain from a state $\sigma$. This is best understood in the context of the axiomatic formulation in [9] (see also [10, 13]), where $\mathcal{B}(\sigma, P)$ can be identified with the (abstract) work function. It is clear from the same axiomatic developments that $\psi_{m}$ is a non-negative quantity.

We denote by $P^{(m)}(\tau)=\left(\mathbf{g}^{(m)}(\tau), \nabla \mathbf{g}^{(m)}(\tau)\right)$ the optimal process, which yields such a maximum. With reference to such a process we consider

$$
\mathbf{g}_{P}(\tau)=\mathbf{g}^{(m)}(\tau)+\delta \mathbf{e}(\tau), \nabla \mathbf{g}_{P}(\tau)=\nabla \mathbf{g}^{(m)}(\tau)+\gamma \mathbf{h}(\tau) \forall \tau \in \mathbf{R}^{+},
$$

where $\mathbf{e}$ and $\mathbf{h}$ are two arbitrary smooth functions, such that $\mathbf{e}(0)=\mathbf{0}, \mathbf{h}(0)=\mathbf{0}$, and $\gamma$ and $\delta$ are two real parameters. 
Substituting (6.11) into the expression $(\underline{6.9})_{2}$ gives

$$
\begin{aligned}
-\mathcal{B}(\sigma, P)= & -\frac{1}{2} \int_{0}^{+\infty} \int_{0}^{+\infty} K_{1}(|\tau-\eta|)\left\{\mathbf{g}^{(m)}(\eta) \cdot \mathbf{g}^{(m)}(\tau)\right. \\
& \left.+\delta\left[\mathbf{g}^{(m)}(\eta) \cdot \mathbf{e}(\tau)+\mathbf{e}(\eta) \cdot \mathbf{g}^{(m)}(\tau)\right]+\delta^{2} \mathbf{e}(\eta) \cdot \mathbf{e}(\tau)\right\} d \eta d \tau \\
& +\int_{0}^{+\infty} \mathbf{I}^{t}\left(\tau, \overline{\mathbf{g}}^{t}\right) \cdot\left[\mathbf{g}^{(m)}(\tau)+\delta \mathbf{e}(\tau)\right] d \tau \\
& -\frac{1}{2} \int_{0}^{+\infty} \int_{0}^{+\infty} K_{2}(|\tau-\eta|)\left\{\nabla \mathbf{g}^{(m)}(\eta) \cdot \nabla \mathbf{g}^{(m)}(\tau)\right. \\
& \left.+\gamma\left[\nabla \mathbf{g}^{(m)}(\eta) \cdot \mathbf{h}(\tau)+\mathbf{h}(\eta) \cdot \nabla \mathbf{g}^{(m)}(\tau)\right]+\gamma^{2} \mathbf{h}(\eta) \cdot \mathbf{h}(\tau)\right\} d \eta d \tau \\
& +\int_{0}^{+\infty} \Im^{t}\left(\tau, \overline{\mathbf{g}}^{t}\right) \cdot\left[\nabla \mathbf{g}^{(m)}(\tau)+\gamma \mathbf{h}(\tau)\right] d \tau,
\end{aligned}
$$

whence, differentiating with respect to $\delta$ and $\gamma$, we have the following system:

$$
\left\{\begin{aligned}
&\left.\frac{\partial}{\partial \delta}[-\mathcal{B}(\sigma, P)]\right|_{\delta=0}=\int_{0}^{+\infty} \mathbf{e}(\tau) \cdot[-\int_{0}^{+\infty} K_{1}(|\tau-\eta|) \mathbf{g}^{(m)}(\eta) d \eta \\
&\left.+\mathbf{I}^{t}\left(\tau, \overline{\mathbf{g}}^{t}\right)\right] d \tau=0 \\
&\left.\frac{\partial}{\partial \gamma}[-\mathcal{B}(\sigma, P)]\right|_{\gamma=0}=\int_{0}^{+\infty} \mathbf{h}(\tau) \cdot\left[-\int_{0}^{+\infty} K_{2}(|\tau-\eta|) \nabla \mathbf{g}^{(m)}(\eta) d \eta\right. \\
&\left.+\mathfrak{I}^{t}\left(\tau, \nabla \overline{\mathbf{g}}^{t}\right)\right] d \tau=0 .
\end{aligned}\right.
$$

This system must be satisfied by arbitrary $\mathbf{e}$ and $\mathbf{h}$; therefore, it follows that

$$
\left\{\begin{array}{l}
\int_{0}^{+\infty} K_{1}(|\tau-\eta|) \mathbf{g}^{(m)}(\eta) d \eta=\mathbf{I}^{t}\left(\tau, \overline{\mathbf{g}}^{t}\right), \\
\int_{0}^{+\infty} K_{2}(|\tau-\eta|) \nabla \mathbf{g}^{(m)}(\eta) d \eta=\mathfrak{I}^{t}\left(\tau, \nabla \overline{\mathbf{g}}^{t}\right),
\end{array} \quad \forall \tau \in \mathbf{R}^{+},\right.
$$

which is a system of two Wiener-Hopf integral equations of the first kind. Using (6.2), we can write it in the form

$$
\left\{\begin{array}{l}
\int_{0}^{+\infty} K_{1}(|\tau-\eta|) \mathbf{g}^{(m)}(\eta) d \eta=-\int_{-\infty}^{0} K_{1}(|\tau-s|) \mathbf{g}^{t}(-s) d s \\
\int_{0}^{+\infty} K_{2}(|\tau-\eta|) \nabla \mathbf{g}^{(m)}(\eta) d \eta=-\int_{-\infty}^{0} K_{2}(|\tau-s|) \nabla \mathbf{g}^{t}(-s) d s
\end{array}\right.
$$

for all $\tau \in \mathbf{R}^{+}$. Let us define

$$
\mathbf{h}_{-}^{t}(\omega)=\int_{-\infty}^{0} \mathbf{g}^{t}(-s) e^{-i \omega s} d s=\left[\mathbf{g}_{+}^{t}(\omega)\right]^{*},
$$

in terms of the notation (7.2). This quantity is analytic in $\mathbf{C}^{+}$. We have

$$
\frac{d}{d t} \mathbf{h}_{-}^{t}(\omega)=i \omega \mathbf{h}_{-}^{t}(\omega)+\mathbf{g}(t) .
$$

It is possible to solve (6.15) and derive the solutions $\mathbf{g}^{(m)}$ and $\nabla \mathbf{g}^{(m)}$, which yield the maximum recoverable action, by virtue of the thermodynamic properties of the kernels and some theorems on factorization. Consequently, by substituting the expressions for $\mathbf{I}^{t}$ and $\mathfrak{I}^{t}$, given by $(\underline{6.14})$, into $(\underline{6.9})_{2}$, we can evaluate this maximum:

$$
\begin{aligned}
\mathcal{B}_{R}(\sigma)= & \frac{1}{2} \int_{0}^{+\infty} \int_{0}^{+\infty} K_{1}(|\tau-\eta|) \mathbf{g}^{(m)}(\eta) \cdot \mathbf{g}^{(m)}(\tau) d \eta d \tau \\
& +\frac{1}{2} \int_{0}^{+\infty} \int_{0}^{+\infty} K_{2}(|\tau-\eta|) \nabla \mathbf{g}^{(m)}(\eta) \cdot \nabla \mathbf{g}^{(m)}(\tau) d \eta d \tau .
\end{aligned}
$$


We can rewrite this expression in terms of Fourier's transform, by applying Plancherel's theorem; we obtain

$$
\begin{aligned}
\mathcal{B}_{R}(\sigma)= & \frac{1}{2 \pi} \int_{-\infty}^{+\infty} K_{1_{c}}(\omega)\left|\mathbf{g}_{+}^{(m)}(\omega)\right|^{2} d \omega \\
& +\frac{1}{2 \pi} \int_{-\infty}^{+\infty} K_{2_{c}}(\omega)\left|\nabla \mathbf{g}_{+}^{(m)}(\omega)\right|^{2} d \omega .
\end{aligned}
$$

It remains to solve the system (6.14). For this purpose, we introduce $\mathbf{r}_{1}(\tau)$ and $\mathbf{r}_{2}(\tau)$, both of which vanish on $\mathbf{R}^{++}$. On $\mathbf{R}^{-}$, they are defined by the fact that they allow us to write (6.14) on $\mathbf{R}$ in the form

$$
\left\{\begin{array}{l}
\int_{0}^{+\infty} K_{1}(|\tau-\eta|) \mathbf{g}^{(m)}(\eta) d \eta=\mathbf{I}^{t}\left(\tau, \overline{\mathbf{g}}^{t}\right)+\mathbf{r}_{\mathbf{1}}(\tau), \\
\int_{0}^{+\infty} K_{2}(|\tau-\eta|) \nabla \mathbf{g}^{(m)}(\eta) d \eta=\mathfrak{I}^{t}\left(\tau, \nabla \overline{\mathbf{g}}^{t}\right)+\mathbf{r}_{\mathbf{2}}(\tau),
\end{array} \quad \forall \tau \in \mathbf{R} .\right.
$$

Using (6.15), we have for all $\tau \in \mathbf{R}$,

$$
\left\{\begin{array}{c}
\int_{0}^{+\infty} K_{1}(|\tau-\eta|) \mathbf{g}^{(m)}(\eta) d \eta \\
\quad=-\int_{-\infty}^{0} K_{1}(|\tau-s|) \mathbf{g}^{t}(-s) d s+\mathbf{r}_{\mathbf{1}}(\tau) \\
\int_{0}^{+\infty} K_{2}(|\tau-\eta|) \nabla \mathbf{g}^{(m)}(\eta) d \eta \\
\quad=-\int_{-\infty}^{0} K_{2}(|\tau-s|) \nabla \mathbf{g}^{t}(-s) d s+\mathbf{r}_{\mathbf{2}}(\tau)
\end{array}\right.
$$

Taking the Fourier transform to these equations, using the notation of (17.2), we have

$$
\left\{\begin{array}{l}
2 K_{1_{c}}(\omega) \mathbf{g}_{+}^{(m)}(\omega)=-2 K_{1_{c}}(\omega) \mathbf{h}_{-}^{t}(\omega)+\mathbf{r}_{1-}(\omega), \\
2 K_{2_{c}}(\omega) \nabla \mathbf{g}_{+}^{(m)}(\omega)=-2 K_{2_{c}}(\omega) \nabla \mathbf{h}_{-}^{t}(\omega)+\mathbf{r}_{2-}(\omega) .
\end{array}\right.
$$

We now consider the function ${ }^{3}$

$$
H_{i}(\omega)=\left(1+\omega^{2}\right) K_{i_{c}}(\omega)>0(i=1,2),
$$

which has no zero for any real $\omega$ and also at infinity, by virtue of (4.24), (4.27) and (4.28). Consequently, we can factorize both $H_{i}(\omega)$ and $K_{i_{c}}(\omega)(i=1,2)$ [15], since

$$
H_{i}(\omega)=H_{i(+)}(\omega) H_{i(-)}(\omega) \equiv(1+i \omega)(1-i \omega) K_{i_{c}}(\omega)(i=1,2),
$$

so that

$$
K_{i_{c}}(\omega)=\frac{H_{i(+)}(\omega)}{(1+i \omega)} \frac{H_{i(-)}(\omega)}{(1-i \omega)} \equiv K_{i(+)}(\omega) K_{i(-)}(\omega)(i=1,2),
$$

where

$$
K_{i( \pm)}(\omega)=\frac{H_{i( \pm)}(\omega)}{1 \pm i \omega}(i=1,2) .
$$

Note that $K_{1( \pm)}(\omega)$ tend to zero as $\omega^{-1}$ for large $\omega$. Also, we have that all the singularities and zeros of $K_{1( \pm)}$ are in $\mathbf{C}^{ \pm}$, respectively, and the factors can be chosen so that

$$
\left[K_{i( \pm)}(\omega)\right]^{*}=K_{i( \pm)}(-\omega)=K_{i(\mp)}(\omega) \quad(i=1,2) .
$$

\footnotetext{
${ }^{3}$ The factor $\left(1+\omega^{2}\right)$ in 6.23 may be $\left(\omega_{0}^{2}+\omega^{2}\right)$, where $\omega$ is any real frequency. We assign it a value of unity for convenience.
} 
Thus, from (6.22), we have

$$
\begin{aligned}
& K_{1(+)}(\omega) \mathbf{g}_{+}^{(m)}(\omega)=-K_{1(+)}(\omega) \mathbf{h}_{-}^{t}(\omega)+\frac{\mathbf{r}_{1-}(\omega)}{2 K_{1(-)}(\omega)}, \\
& K_{2(+)}(\omega) \nabla \mathbf{g}_{+}^{(m)}(\omega)=-K_{2(+)}(\omega) \nabla \mathbf{h}_{-}^{t}(\omega)+\frac{\mathbf{r}_{2-}(\omega)}{2 K_{2(-)}(\omega)} .
\end{aligned}
$$

By virtue of the Plemelj formulae [21, it follows that

$$
\begin{aligned}
K_{1(+)}(\omega) \mathbf{h}_{-}^{t}(\omega) & =\mathbf{P}_{(1)(-)}^{t}(\omega)-\mathbf{P}_{(1)(+)}^{t}(\omega), \\
K_{2(+)}(\omega) \nabla \mathbf{h}_{-}^{t}(\omega) & =\mathbf{P}_{(2)(-)}^{t}(\omega)-\mathbf{P}_{(2)(+)}^{t}(\omega),
\end{aligned}
$$

where

$$
\begin{aligned}
\mathbf{P}_{(1)}^{t}(z) & =\frac{1}{2 \pi i} \int_{-\infty}^{+\infty} \frac{K_{1(+)}(\omega) \mathbf{h}_{-}^{t}(\omega)}{\omega-z} d \omega, \\
\mathbf{P}_{(1)( \pm)}^{t}(\omega) & =\lim _{\beta \rightarrow 0 \mp} \mathbf{P}_{(1)}^{t}(\omega+i \beta), \\
\mathbf{P}_{(2)}^{t}(z) & =\frac{1}{2 \pi i} \int_{-\infty}^{+\infty} \frac{K_{2(+)}(\omega) \nabla \mathbf{h}_{-}^{t}(\omega)}{\omega-z} d \omega, \\
\mathbf{P}_{(2)( \pm)}^{t}(\omega) & =\lim _{\beta \rightarrow 0 \mp} \mathbf{P}_{(2)}^{t}(\omega+i \beta) .
\end{aligned}
$$

Using (6.29) in (6.28), we obtain

$$
\begin{aligned}
\mathbf{U}(\omega) & \equiv K_{1(+)}(\omega) \mathbf{g}_{+}^{(m)}(\omega)-\mathbf{P}_{(1)(+)}^{t}(\omega) \\
& =-\mathbf{P}_{(1)(-)}^{t}(\omega)+\frac{\mathbf{r}_{1-}(\omega)}{2 K_{1(-)}(\omega)}, \\
\mathbf{V}(\omega) & \equiv K_{2(+)}(\omega) \nabla \mathbf{g}_{+}^{(m)}(\omega)-\mathbf{P}_{(2)(+)}^{t}(\omega) \\
& =-\mathbf{P}_{(2)(-)}^{t}(\omega)+\frac{\mathbf{r}_{2-}(\omega)}{2 K_{2(-)}(\omega)} .
\end{aligned}
$$

The two functions $\mathbf{U}$ and $\mathbf{V}$ are defined by means of two different expressions, which, considered as functions of $z \in \mathbf{C}$, are analytic in $\mathbf{C}^{-}$and analytic in $\mathbf{C}^{+}$, respectively; they also vanish at infinity. Therefore, both of them must be equal to zero and we obtain the solutions

$$
\mathbf{g}_{+}^{(m)}(\omega)=\frac{\mathbf{P}_{(1)(+)}^{t}(\omega)}{K_{1(+)}(\omega)}, \quad \nabla \mathbf{g}_{+}^{(m)}(\omega)=\frac{\mathbf{P}_{(2)(+)}^{t}(\omega)}{K_{2(+)}(\omega)},
$$

which allow us to write (6.10), using (6.19) and (6.32), as follows:

$$
\begin{aligned}
\psi_{m}(t) \equiv \mathcal{B}_{R}(\sigma)=\frac{1}{2 \pi} \int_{-\infty}^{+\infty}\left|\mathbf{P}_{(1)(+)}^{t}(\omega)\right|^{2} d \omega \\
+\frac{1}{2 \pi} \int_{-\infty}^{+\infty}\left|\mathbf{P}_{(2)(+)}^{t}(\omega)\right|^{2} d \omega
\end{aligned}
$$

REMARK 6.1. Comparing this expression with the results of [14, 6, 10] and other references, one observes that $\mathbf{P}_{(i)(+)}^{t}(i=1,2)$ occur with singularities in $\mathbf{C}^{(+)}$rather than functionals similarly defined but with singularities in $\mathbf{C}^{(-)}$. Note however that the results of [13] and other papers agree with (6.33). The source of this difference is 
simply that histories and continuations used here are time-reversed compared with those in earlier works, leading to complex conjugates in the frequency domain, which of course leaves quantities of the form (6.33) unchanged.

We now express (4.15) in terms of Fourier domain quantities. Parseval's formula yields

$$
\begin{aligned}
\mathcal{A}(t)=\frac{1}{\pi} & \int_{-\infty}^{+\infty} K_{1 c}(\omega) \mathbf{h}_{-}^{t}(\omega) d \omega \cdot \mathbf{g}(t) \\
& +\frac{1}{\pi} \int_{-\infty}^{+\infty} K_{2 c}(\omega) \nabla \mathbf{h}_{-}^{t}(\omega) d \omega \cdot \nabla \mathbf{g}(t) .
\end{aligned}
$$

Now, from (6.29),

$$
\begin{aligned}
& \frac{1}{\pi} \int_{-\infty}^{+\infty} K_{1 c}(\omega) \mathbf{h}_{-}^{t}(\omega) d \omega \\
& \quad=\frac{1}{\pi} \int_{-\infty}^{+\infty} K_{1(-)}(\omega)\left[\mathbf{P}_{(1)(-)}^{t}(\omega)-\mathbf{P}_{(1)(+)}^{t}(\omega)\right] d \omega \\
& \quad=-\frac{1}{\pi} \int_{-\infty}^{+\infty} K_{1(-)}(\omega) \mathbf{P}_{(1)(+)}^{t}(\omega) d \omega
\end{aligned}
$$

since the term involving $\mathbf{P}_{(1)(-)}^{t}(\omega)$ vanishes by closing the contour on $\mathbf{C}^{(+)}$. A similar result applies to the second integral in (6.34). The last form of (6.35) and the corresponding result for the second integral must be real, since $\mathcal{A}$ is real.

Finally, we seek to determine the rate of dissipation associated with $\psi_{m}(t)$. Following the steps outlined in [14] and other papers, we find, with the aid of (6.17), that

$$
\begin{aligned}
\frac{d}{d t} \mathbf{P}_{(1)(+)}^{t}(\omega) & =i \omega \mathbf{P}_{(1)(+)}^{t}(\omega)+\mathbf{K}_{1}(t)-K_{1(+)}(\omega) \mathbf{g}(t) \\
\frac{d}{d t} \mathbf{P}_{(1)(-)}^{t}(\omega) & =i \omega \mathbf{P}_{(1)(-)}^{t}(\omega)+\mathbf{K}_{1}(t) \\
\mathbf{K}_{1}(t) & =\frac{1}{2 \pi} \int_{-\infty}^{+\infty} K_{1(+)}(\omega) \mathbf{h}_{-}^{t}(\omega) d \omega
\end{aligned}
$$

The time derivatives of $\mathbf{P}_{(2)( \pm)}^{t}$ yield similar expressions but with $\mathbf{g}(t)$ replaced by $\nabla \mathbf{g}(t)$ and $\mathbf{K}_{1}(t)$ replaced by $\mathbf{K}_{2}(t)$, where

$$
\mathbf{K}_{2}(t)=\frac{1}{2 \pi} \int_{-\infty}^{+\infty} K_{2(+)}(\omega) \nabla \mathbf{h}_{-}^{t}(\omega) d \omega .
$$

Also,

$$
\frac{1}{2 \pi} \int_{-\infty}^{+\infty} \mathbf{P}_{(i)(\mp)}^{t}(\omega) d \omega= \pm \frac{1}{2} \mathbf{K}_{i}(t), \quad i=1,2 .
$$

Differences from earlier results relate to Remark 6.1 and the fact that $K_{1( \pm)}(\omega)$ vanish at large $\omega$. The complex conjugate relations are easily obtained on noting (6.27).

If we differentiate (6.33) and use these relations, then on recalling (4.12), (6.34) and 6.35), we find that

$$
\begin{aligned}
D_{m}(t) & =\left|\mathbf{K}_{1}(t)\right|^{2}+\left|\mathbf{K}_{2}(t)\right|^{2} \\
& =\mathbf{K}_{1}^{2}(t)+\mathbf{K}_{2}^{2}(t)
\end{aligned}
$$


since the $\mathbf{K}_{i}, i=1,2$ are real; this follows by taking the complex conjugate of $(6.36)_{3}$, (6.37) and changing the integration variables.

7. Appendix. The Fourier transform of any function $f: \mathbf{R} \rightarrow \mathbf{R}^{n}$ is defined by

$$
f_{F}(\omega)=\int_{-\infty}^{+\infty} f(s) e^{-i \omega s} d s=f_{-}(\omega)+f_{+}(\omega) \quad \forall \omega \in \mathbf{R},
$$

where

$$
f_{-}(\omega)=\int_{-\infty}^{0} f(s) e^{-i \omega s} d s, \quad f_{+}(\omega)=\int_{0}^{+\infty} f(s) e^{-i \omega s} d s
$$

The half-range Fourier cosine and sine transforms are given by

$$
f_{c}(\omega)=\int_{0}^{+\infty} f(s) \cos \omega s d s, \quad f_{s}(\omega)=\int_{0}^{+\infty} f(s) \sin \omega s d s
$$

they hold even if $f$ is defined only on $\mathbf{R}^{+}$, as it occurs for $f_{+}$.

Any function $f$ defined only on $\mathbf{R}^{+}$can be extended on $\mathbf{R}$ in several ways. We recall the following expressions:

$$
2 f_{F}(\omega)=f_{c}(\omega)-i f_{s}(\omega), \quad f_{F}(\omega)=2 f_{c}(\omega), \quad f_{F}(\omega)=-2 i f_{s}(\omega)
$$

for the Fourier transform of the new functions corresponding to the extension made with the causal extension, $f(\xi)=0 \forall \xi<0$, or with an even function, $f(\xi)=f(-\xi) \forall \xi<0$, or with an odd one, $f(\xi)=-f(-\xi) \forall \xi<0$, respectively.

Moreover, if also $f$ and $f^{\prime}$ belong to $L^{1}\left(\mathbf{R}^{+}\right) \cap L^{2}\left(\mathbf{R}^{+}\right)$, we have

$$
f_{s}^{\prime}(\omega)=-\omega f_{c}(\omega)
$$

If $f^{\prime}(0)$ is non-zero, we have the important relation

$$
\lim _{\omega \rightarrow \infty} i \omega f_{+}^{\prime}(\omega)=f^{\prime}(0)=\lim _{\omega \rightarrow \infty} \omega f_{s}^{\prime}(\omega)=-\lim _{\omega \rightarrow \infty} \omega^{2} f_{c}(\omega),
$$

by using (7.5).

Finally, we denote by $\mathbf{C}^{(\mp)}$ and $\mathbf{C}^{\mp}$ the subsets of the complex $z$-plane $\mathbf{C}$, defined by

$$
\mathbf{C}^{(-)}=\left\{z \in \mathbf{C} ; \operatorname{Im} z \in \mathbf{R}^{--}\right\}, \quad \mathbf{C}^{(+)}=\left\{z \in \mathbf{C} ; \operatorname{Im} z \in \mathbf{R}^{++}\right\},
$$

where $\mathbf{R}^{--}=(-\infty, 0)$ and $\mathbf{R}^{++}=(0,+\infty)$ and

$$
\mathbf{C}^{-}=\left\{z \in \mathbf{C} ; \operatorname{Im} z \in \mathbf{R}^{-}\right\}, \quad \mathbf{C}^{+}=\left\{z \in \mathbf{C} ; \operatorname{Im} z \in \mathbf{R}^{+}\right\} .
$$

We observe that $f_{ \pm}(\omega)$, given by (17.2), can be extended in $\mathbf{C}$ and become analytic functions in $\mathbf{C}^{(\mp)}$. On assuming the analyticity of the Fourier transforms on $\mathbf{R}\left[14, f_{ \pm}(z)\right.$ become analytic in the subsets $\mathbf{C}^{\mp}$, which in fact include the real axis. The notation $f_{( \pm)}(z)$ is used in general (not necessarily in the context of Fourier transforms) to denote that the singularities of $f$ are only in $\mathbf{C}^{ \pm}$. 


\section{REFERENCES}

[1] G. Amendola, A. Manes and C. Vettori, Maximum recoverable work for a rigid heat conductor with memory, Acta Appl. Math. 110 (3) (2010), 1011-1036. MR2639155 (2011e:80004)

[2] G. Amendola and S. Carillo, Thermal work and minimum free energy in a heat conductor with memory, Quart. J. Mech. Appl. Math. 57 (3) (2004), 429-446. MR2088844(2005f:80002)

[3] C. Cattaneo, Sulla conduzione del calore, Atti Sem. Mat. Fis. Univ. Modena 3 (1948), 83-101. MR0032898 (11:362d)

[4] V. A. Cimmelli and K. Frischmuth, Gradient generalization to the extended thermodynamic approach and diffusive-hyperbolic heat conduction, Physica B: Condensed Matter (1-2) 400 (2007), 257-265.

[5] B.D. Coleman, Thermodynamics of materials with memory, Arch. Rational Mech. Anal. 17 (1964), 1-46. MR0171419 (30:1650)

[6] L. Deseri, M. Fabrizio and J.M. Golden, The concept of minimal state in viscoelasticity: new free energies and applications to PDEs, Arch. Rational Mech. Anal. 181(1) (2006), 43-96. MR2221203 (2009a:74024)

[7] M. Fabrizio, Free energies in the materials with fading memory and applications to PDEs, Proceedings WASCOM 2003, World Sci., Singapore, 2004. MR2089846

[8] M. Fabrizio, G. Gentili and D.W. Reynolds, On rigid heat conductors with memory, Int. J. Engrg. Sci. 36 (1998), 765-782. MR1629806 (99i:80006)

[9] M. Fabrizio, C. Giorgi and A. Morro, Free energies and dissipation properties for systems with memory, Arch. Rational Mech. Anal. 125 (1994), 341-373. MR.1253168 (95j:73012)

[10] M. Fabrizio and J.M. Golden, Maximum and minimum free energies for a linear viscoelastic material, Quart. Appl. Math. LX (2) (2002), 341-381. MR1900497(2003b:74013)

[11] M. Fabrizio and B. Lazzari, On asymptotic stability for linear viscoelastic fluids, Diff. Int. Eq., 6 (3) (1993), 491-504. MR.1202554 (94b:76009)

[12] M. Fabrizio and A. Morro, Mathematical problems in linear viscoelasticity, SIAM Studies in Applied Mathematics, Philadelphia, 1992. MR1153021 (93a:73034)

[13] G. Gentili, Maximum recoverable work, minimum free energy and state space in linear viscoelasticity, Quart. Appl. Math. LX (1) (2002), 153-182. MR.1878264 (2002m:74011)

[14] J.M. Golden, Free energy in the frequency domain: The scalar case, Quart. Appl. Math. LVIII (1) (2000), 127-150. MR 1739041 (2001e:74025)

[15] L. Deseri, G. Gentili, J. M. Golden, An explicit formula for the minimum free energy in linear viscoelasticity, J. Elasticity 54 (1999) 141-185. MR1728444(2001i:74012)

[16] D. Graffi, Sull'espressione analitica di alcune grandezze termodinamiche nei materiali con memoria, Rend. Sem. Mat. Univ. Padova, 68 (1982), 17-29.

[17] D. Graffi, Ancora sull'espressione dell'energia libera nei materiali con memoria, Atti Acc. Scienze Torino, 120 (1986), 111-124. MR958166 (90f:73030)

[18] M.E. Gurtin and A.C. Pipkin, A general theory of heat conduction with finite wave speeds, Arch. Rational Mech. Anal. 31 (1968), 113-126. MR.1553521

[19] R. A. Guyer and J. A. Krumhansl, Solution of the linearized Phonon Boltzmann equation, Phys. Rev. 148 (1966), 766-778.

[20] I. Müller, On the entropy inequality, Arch. Rational Mech. Anal. 26 (1967), 118-141. MR0214336 $(35: 5187)$

[21] N.I. Muskhelishvili, Singular Integral Equations, Noordhoff, Groningen, 1953. MR0355494|(50:7968)

[22] V. Volterra, Theory of functional and of integral and integro-differential equations, Blackie \& Son Limited, London, 1930. 